\title{
The Study and Implementation of Agricultural Information Service System Based on Addressable Broadcast
}

\author{
Huoguo Zheng ${ }^{1,2}$, Haiyan $\mathrm{Hu}^{1,2}$, Shihong $\mathrm{Liu}^{1,2}$, and Hong Meng ${ }^{1,2}$ \\ ${ }^{1}$ Key Laboratory of Digital Agricultural Early-warning Technology Ministry of Agriculture, \\ People's Republic of China, Beijing 100081, P.R. China \\ ${ }^{2}$ Agricultural Information Institute of Chinese Academy of Agriculture Science, \\ Beijing 100081, P.R. China
}

\begin{abstract}
The broadcast population coverage has increased to $96 \%$ in china. Information services by radio have wide coverage, low cost, easy to grass-roots farmers to accept etc. characteristics. In order to play the better role of broadcast information service in rural area, the author R \& D agricultural information service system based on addressable broadcast, which transformed from traditional broadcast with a set of data encoding/decoding equipments based on addressable broadcast. The agricultural information service system based on addressable broadcast can provide personalized information services for grassroots farmers, relying on a wealth of agricultural information resources, by means of controlling the addressable speakers with microprocessor. The system has five parts, which has been applied in huailai city Hebei province. The system has provided personalized information about grape and vegetable for two villages for one and a half years.
\end{abstract}

Keywords: agricultural information service system, addressable broadcast, agriculture, economic growth, panel data, regression.

\section{Introduction}

After several years of development, China has made the amazing progress in the development face the farmer's information service. Agricultural information service played a more positive role in solving the problem of "Farmers, Rural Areas and Agriculture Production" [1]. In the recently years, there had emerged a number of new agriculture information service patterns, which can provide timely and accurate information for farmers by the media of TV, phone and network[2]. However, we should see that the radio and TV are still playing the main role of information service infrastructure in rural. In fact, these information services mode use efficiency is very low, because the grass-roots farmers' lack of the motive due to the absence of search and use the information actively[3]. According to the latest statistics, China's radio and television coverage has increased to more than $98 \%$. Compared with other services, broadcasting as a carrier of information services has a wide coverage and low cost, 
easy-to-grass-roots farmers to accept and so on. On the other hand, grass-roots farmers can access the information at all times whether they willing or not, because broadcast information service running in passive mode.

After the investigation and study we discovered that broadcast information service emerged quietly in many places and the prospects for development are huge[4]. To develop the broadcast information service well, we have researched and developed the rural broadcast information service system based on addressable broadcast in this paper, which can provide personalized information service.

\section{Principles and Type of Addressable Broadcast}

\subsection{The Principle of Addressable Broadcast}

Based on the existing FM radio signal transmission system and with the addressable control technology, addressable broadcast can control any one FM speaker's FM receive frequencies, switch status and volume status individually in service area. In other words, addressable broadcast achieve point to point and multi-function control.

Addressable broadcast uses the main channel of FM radio transmission audio signals, meanwhile, uses the sub-channel of FM radio transmission control information, which also known as FM-SCA addressable control system [5].

The core of addressable broadcast is FM sub-channel wireless addressable system, which is also called wireless addressable control system. By means of the wireless control technology of FM-SCA (Frequency Modulation-Subsidiary Communication's Authorizations), which transmit the addressing/controlling signal to the speakers through FM sub-channel in SCA way, wireless addressable system can control the exact speaker of the service area.

Addressable control technology of FM-SCA achieves the function of wireless control broadcast with the help of FSK (Frequency Shift Keying) in FM sub-channel. The existing FM radio only used to send signal of broadcast. As the baseband signal bandwidth of FM stereo is $53 \mathrm{kHz}$, the baseband signal bandwidth of FM mono radio is $53 \mathrm{kHz}$, so the bandwidth from $53 \mathrm{kHz}$ to $100 \mathrm{kHz}$ or from $15 \mathrm{kHz}$ to $100 \mathrm{kHz}$ in FM radio almost idle. There are several sub-channel are available for use in each frequency channel. In china, $67 \mathrm{kHz}$ is used for sub-channel[6].

\section{Transmitting principle of FM-SCA wireless addressable control system}

The format of baseband signal source coding of FM-SCA wireless addressable control system is Manchester, and the technology of modulation is FSK. So, if some software

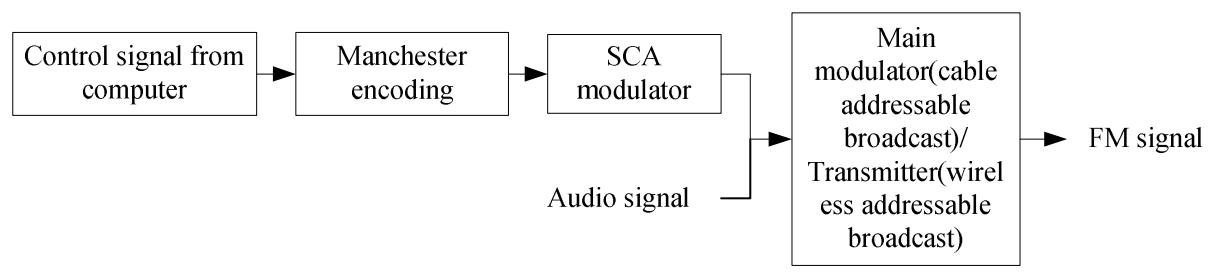

Fig. 1. Transmitting principle of FM-SCA 
and hardware are added to the original FM radio, we just achieve addressable broadcast. The transmitting principle of FM-SCA wireless addressable control system is shown in Fig. 1.

At the sending end, addressing control information is encoded by way of Manchester, which must meet the energy diffusion theory in order to send reliably. SCA modulator modulates the data that has been transformed by Manchester method in the way of FSK. In other words, the required sending data are modulated to a frequency of $67 \mathrm{kHz}$ sub-carrier in the way of FSK, and then $67 \mathrm{kHz}$ sub-carrier signals are added to the main FM tuner.

\section{Receiving principle of FM-SCA wireless addressable control system}

When FM radio signals are received by antenna, after four steps, these signals are transformed into composite baseband signals, which include two kinds of signals: audio signal and addressing control signal. First, these signals are amplified by low-noise amplifier; second, these signals are mixed with a frequency of $10.7 \mathrm{kHz}$; third, these signals are filtered by BF Band filter; in the fourth step, these signals are amplified by intermediate frequency amplifier and frequency discriminator. Finally, composite baseband signals are worked out. FM radio receiver obtain audio signal by means of filtering those $53 \mathrm{kHz}$ above signals with low pass filter. Meanwhile, SCA receiver gets $67 \mathrm{kHz}$ addressing control information through $\mathrm{BF}$ Band filter after these signals are handled by frequency discriminator. These addressing control signals can control speaker's FM receive frequencies, switch status and volume status with a series of processing. The receiving principle of FM-SCA wireless addressable control system is shown in Fig. 2.

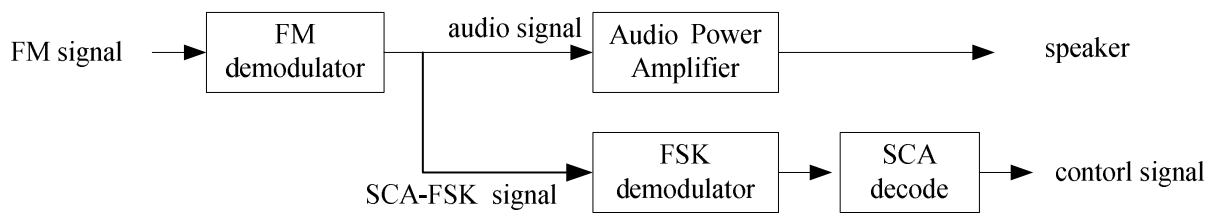

Fig. 2. Receiving principle of FM-SCA

\subsection{Type of Addressable Broadcast}

There are two kinds of addressable broadcast: cable addressable broadcast and wireless addressable broadcast. Cable addressable broadcast is constructed based on the CATV FM common cable transmission system, which suits for the area of CATV network coverage over and not self-built radio station. Wireless addressable broadcast suits for the county or city which has a radio station.

Cable addressable broadcast has four main parts: control software in computer, data encoder, modulation host and addressable FM speaker. The work flow of cable addressable broadcast is described below: User's instructions are received by controlling software in computer, and then these instructions are sent to data encoder through native COM port with specific format. The addressing control signals are 
changed to FM sub-channel by data encoder, and then sent to FM modulator together with audio signals. Those mixed signals on some RF carrier are sent to addressable FM speaker through the existing CATV network. The work flow of cable addressable broadcast is shown in Fig. 3.

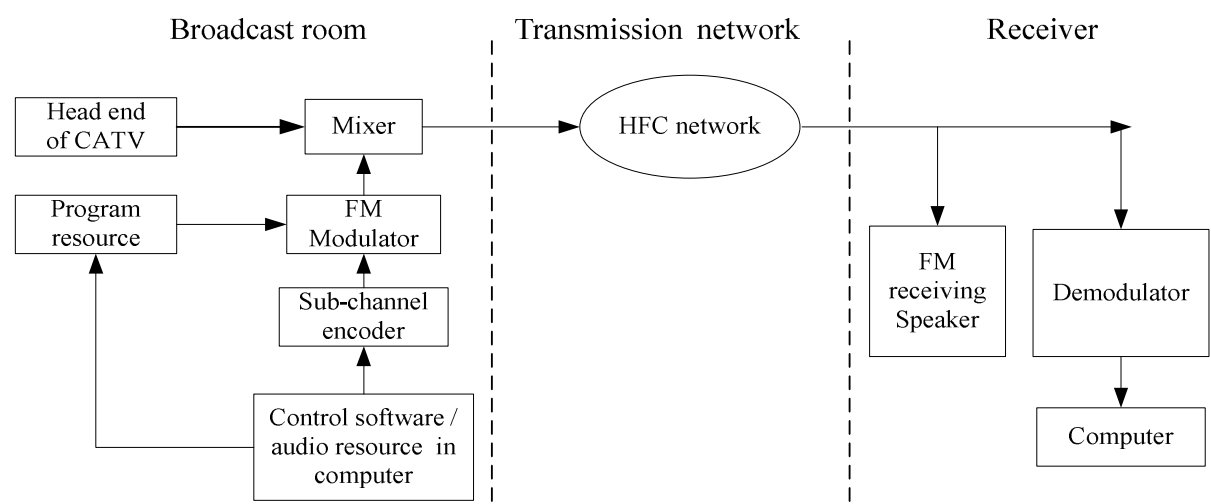

Fig. 3. Work flow of cable addressable broadcast

Based on the existing wireless radio broadcast, with the help of FM-SCA, wireless addressable broadcast can work. The work flow of wireless addressable broadcast is: User's instructions received by controlling software are sent to sub-channel encoder, and then sent by transmitter mixed with audio signals. The work flow of cable addressable broadcast is shown in Fig. 4.

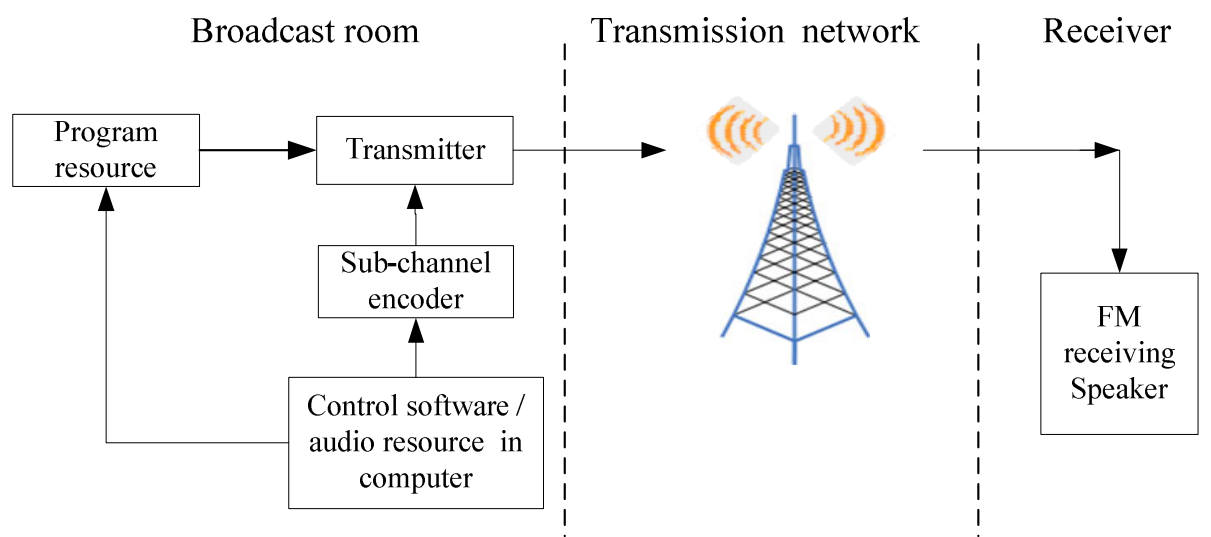

Fig. 4. Work flow of wireless addressable broadcast 


\section{Design of Agricultural Information Service System Based on Addressable Broadcast}

\subsection{Structure of Agricultural Information Service System}

The work flow of agricultural information service system based on addressable broadcast is described as follow: (1) if farmers want to get some information, they tell the radio station their requirements by means of telephone or SMS;(2) radio station get the information and then edit program once receiving the requirements from farmers; 3 ) when the program prepared, they will broadcast by the addressable broadcast system, (4)and farmers can listen to the program with the addressing radio receiver. Fig. 5 shows the service flow of the system.

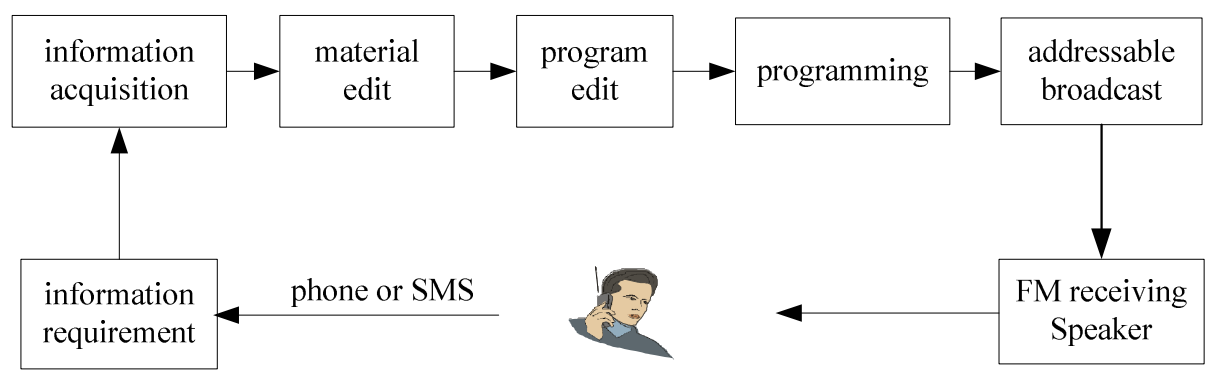

Fig. 5. Work flow of agricultural information service system

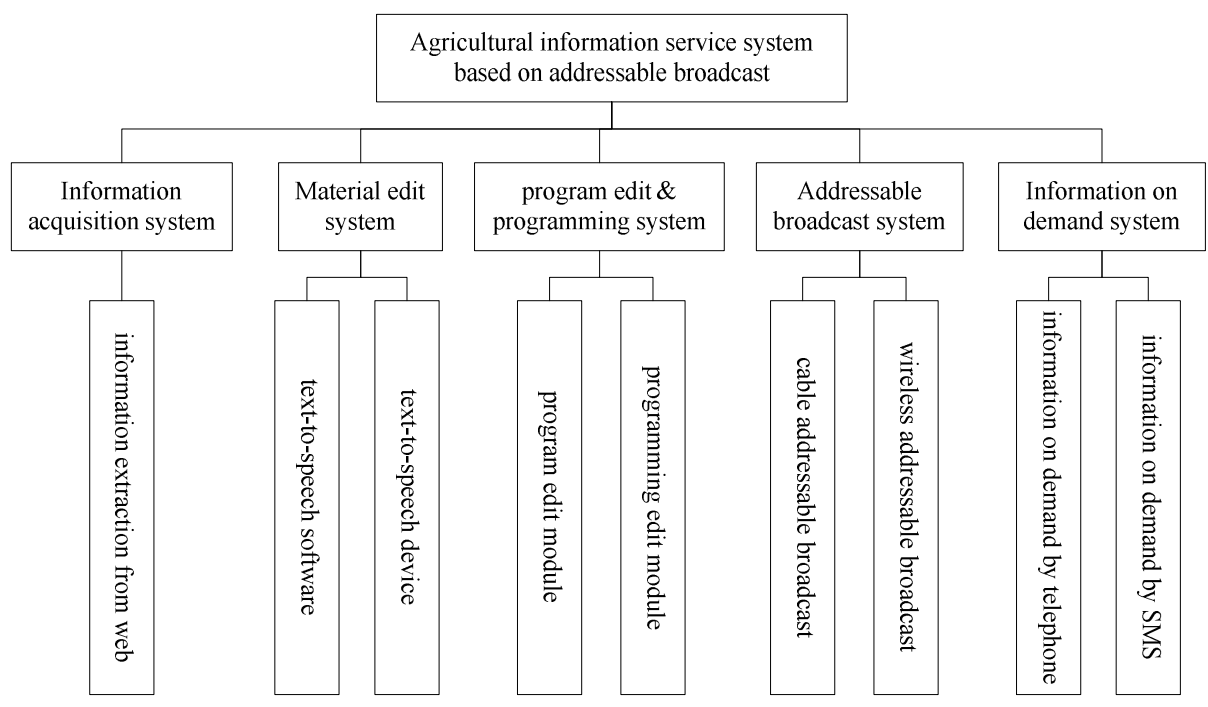

Fig. 6. Function diagram of agricultural information service system 
Agricultural information service system based on addressable broadcast has five sub-systems: information acquisition system, material edit system, program edit \& programming system, addressable broadcast system and information on demand system. The function diagram of agricultural information service system is shown in Fig. 6.

\subsection{Function of Agricultural Information Service System}

With agricultural information service system based on addressable broadcast, we can realize personalized information service. Different information needs in different regions can be satisfied by means of broadcasting different programs at the same time.

The function of five sub-systems is described as below:

(1) Information acquisition system

Information can be extracted from internet according to the requirements from farmers by the information acquisition system. The system can extract information automatically in accordance with pre-set; also can get the information specified by user.

(2) Material edit system

In material edit system, the information (text formatting) extracted from information acquisition system can be transferred into audio file through the text-to-speech software or the text-to-speech device. Those audio file generated by this system will be used in program edit module.

(3) Program edit \& programming system

In this system, audio file generated by material edit module will be made into program, and then broadcast the program at a particular time with the radio equipment in radio station.

(4) Addressable broadcast system

Addressable broadcast system is the core of the whole system, which make the personalized information service possible. With the FM-SCA technology of addressable broadcast system, radio station can make different area or different speaker broadcast different program.

(5) Information on demand system

If listeners want to get some information that radio station don't provide, they can tell the radio station their personalized information requirements through information on demand system. There are two ways to express the needs for listeners: telephone or SMS.

\section{Conclusion}

In this paper, the principles and type of addressable broadcast is introduced, which uses the sub-channel of FM radio transmission control information. Based on the addressable broadcast, agricultural information service system is constructed in radio station, which concludes five parts. The system can provide personalized information service for farmers in rural area. The advantages of the system are concluded as follows: 
(1) Agricultural information service system based on addressable broadcast is a new information service mode, which suit for rural area. As we know, the coverage of radio if very high in china, so this system has a very large scope of use.

(2) It is very easy to construct the addressable broadcast system. If there already has a radio station, we just need several equipments.

(3) With information acquisition system, radio station can get huge amounts of information; also can get the exact information meet the listener. The text formatting information can be transferred into audio files through text-to-speech module of material edit system.

Acknowledgements. This study is supported by The National High Technology Research and Development Program of China called as "863" (2007AA10Z236) and The Special Fund for Basic Research Work of Central Scientific Research Institution (2010-J-02).

\section{References}

1. Guo, H., Pan, Y.: Agriculture information service practice and discussion. Agricultural network Information 8(3) (2007)

2. Gao, J., Liu, Y., Cui, J.: The "three electricity and a hall” Information service system help the farmer be rich. China agricultural information (11) (2005)

3. Jilin farmer website. 12316 new rural hotline resolve famer's problem. Jilin agricultural and rural economic information (7) (2007)

4. Chen, X.: Addressable Broadcasting System Plan in Broadcast to Every Village Project. China Digital Cable TV 8, 870-872 (2008)

5. Zheng, Z., Wu, X.: FM addressable broadcast in rural application. Southeast Communication 7, 33-34 (2006)

6. Wang, T.: Application and development of intellectual addressing broadcast system. Sci/tech Information Development \& Economy 2, 251-252 (2005) 